\title{
In situ detection of GM1 and GM2 gangliosides using immunohistochemical and immunofluorescent techniques for auxiliary diagnosis of canine and feline gangliosidoses
}

\author{
Moeko Kohyama ${ }^{1}$, Akira Yabuki ${ }^{1}$, Kenji Ochiai ${ }^{2}$, Yuya Nakamoto ${ }^{3}$, Kazuyuki Uchida ${ }^{4}$, Daisuke Hasegawa ${ }^{5}$, \\ Kimimasa Takahashi ${ }^{6}$, Hiroaki Kawaguchi ${ }^{7}$, Masaya Tsuboi ${ }^{4}$ and Osamu Yamato ${ }^{1 *}$
}

\begin{abstract}
Background: GM1 and GM2 gangliosidoses are progressive neurodegenerative lysosomal storage diseases resulting from the excessive accumulation of GM1 and GM2 gangliosides in the lysosomes, respectively. The diagnosis of gangliosidosis is carried out based on comprehensive findings using various types of specimens for histological, ultrastructural, biochemical and genetic analyses. Therefore, the partial absence or lack of specimens might have resulted in many undiagnosed cases. The aim of the present study was to establish immunohistochemical and immunofluorescent techniques for the auxiliary diagnosis of canine and feline gangliosidoses, using paraffinembedded brain specimens stored for a long period.
\end{abstract}

Results: Using hematoxylin and eosin staining, cytoplasmic accumulation of pale to eosinophilic granular materials in swollen neurons was observed in animals previously diagnosed with GM1 or GM2 gangliosidosis. The immunohistochemical and immunofluorescent techniques developed in this study clearly demonstrated the accumulated material to be either GM1 or GM2 ganglioside.

Conclusions: Immunohistochemical and immunofluorescent techniques using stored paraffin-embedded brain specimens are useful for the retrospective diagnosis of GM1 and GM2 gangliosidoses in dogs and cats.

Keywords: Gangliosidosis, Dog, Cat, Lysosomal Storage Disease, Immunohistochemistry, Immunofluorescence

\section{Background}

GM1 and GM2 gangliosidoses are progressive neurodegenerative lysosomal storage diseases resulting mainly from the excessive accumulation of GM1 and GM2 gangliosides in the lysosomes, respectively [1]. These diseases are inherited in an autosomal recessive manner and result in the premature death of affected individuals due to brain damage with progressive neurological signs. In GM1 gangliosidosis, the accumulation of GM1 ganglioside is caused

\footnotetext{
* Correspondence: osam@vet.kagoshima-u.ac.jp

${ }^{1}$ Laboratory of Clinical Pathology, Department of Veterinary Medicine, Joint Faculty of Veterinary Medicine, Kagoshima University, 1-21-24 Kohrimoto, Kagoshima-shi, Kagoshima 890-0065, Japan

Full list of author information is available at the end of the article
}

by an inherited deficiency of the lysosomal acid $\beta$ galactosidase [2]. In GM2 gangliosidosis, the accumulation of GM2 ganglioside is caused by an inherited deficiency of the lysosomal acid $\beta$-hexosaminidase A or GM2 activator protein in GM2 gangliosidosis, and the disease is accordingly categorized into three variants: Tay-Sachs disease (B variant), Sandhoff disease (0 variant), and GM2 activator protein deficiency (AB variant) [3].

Gangliosidosis is more likely to occur in many animal species and breeds compared to other lysosomal diseases. Naturally occurring GM1 gangliosidosis has been reported in dogs, including mixed Beagles [4], English Springer Spaniels [5], Portuguese Water dogs [6], Alaskan Huskies [7], Shiba Inus [8], and a mixed- 
breed dog [9], and in cats, including Siamese [10, 11], Korat [12], and several families of domestic cats [13-17]. In addition, GM1 gangliosidosis has been reported in ruminants such as Friesian calves [17, 18], Suffolk sheep [19], Coopworth Romny-cross sheep [20], and Romny sheep [21], and in wild species such as American black bears [22] and emus [23]. Naturally occurring GM2 gangliosidosis has been reported in dogs, including German Shorthair Pointers [24], Japanese Spaniels (Chins) [25, 26], a Golden Retriever [27], Toy Poodles [28], and mixed-breed dogs [29,30], and in cats, including Korat [31], European Burmese [32], and several families of domestic cats [33-35]. In addition, GM2 gangliosidosis has been reported in Yorkshire pigs [36], Jacob sheep [37], a rabbit [38], Muntjak deer [39], and American flamingos [40].

The diagnosis of GM1 and GM2 gangliosidoses is carried out based on comprehensive findings, which include clinical, biochemical, histopathological, and genetic findings using various types of specimens $[2,3]$. The clinical findings are progressive neurological, motor, and visual dysfunctions, but they are not specific to these diseases [41]. The biochemical findings include the cerebral accumulation of specific glycoconjugates and deficiency of specific enzyme activities, which are determined by specialized techniques such as thin-layer chromatography (TLC) and fluorometric enzymatic assays, respectively, using fresh or frozen tissues [42, 43]. The histopathological and ultrastructural findings demonstrate swollen neurons filled with periodic acid-Schiff stain-positive storage materials and osmiophilic membranous cytoplasmic bodies in the lysosomes of neurons, respectively, but these characteristics are not completely specific to these diseases $[8,28,30,34]$. Genetic tests can be used to directly diagnose the diseases, but they are limited to diseases for which specific mutations have been identified [44-46]. Therefore, it is possible that a correct diagnosis has not been established in many animal cases, as a result of the partial absence or lack of specimens for biochemical, histological, ultrastructural, or genetic examination.

The aim of the present study was to establish immunohistochemical and immunofluorescent techniques for the auxiliary diagnosis of canine and feline gangliosidoses using paraffin-embedded brain specimens, which are often stored for a long time in veterinary diagnostic laboratories worldwide.

\section{Methods}

\section{Specimens}

Stored paraffin-embedded cerebral cortex samples of dogs and cats with GM1 or GM2 gangliosidosis were used in this study. These cases occurred in different parts of Japan and the original diagnosis was made using specific genetic tests and biochemical analyses at the
Laboratory of Clinical Pathology, Joint Faculty of Veterinary Medicine, Kagoshima University, which has been exclusively supporting the diagnosis of inherited metabolic diseases in animals in Japan. These animals included a 14month-old Shiba Inu with GM1 gangliosidosis diagnosed in 2009, an 11-month-old domestic shorthair cat with GM1 gangliosidosis diagnosed in 2004, a 20-month-old Toy Poodle with GM2 gangliosidosis diagnosed in 2006, and a 20-month-old domestic shorthair cat with GM2 gangliosidosis diagnosed in 2010. The diagnosis of these animals was established using genetic and/or biochemical tests reported previously [11, 43-45]. Stored paraffinembedded cerebral cortex samples of a dog and a cat without any brain disease were also used as controls. Thin sections at $4 \mu \mathrm{m}$ were prepared from these paraffinembedded tissue blocks by standard method. These sections were stained with hematoxylin and eosin (HE) and subjected to the immunohistochemical and immunofluorescent techniques described below. All experimental procedures and ethical issues involving animals and their samples were approved by the the Animal Research Committee at Kagoshima University with the approval number VM15041.

\section{Immunohistochemical study}

Each section was deparaffinized with xylene and rehydrated through a graded ethanol series. Antigen retrieval was conducted by heating the sample in a $10 \mathrm{mM}$ citrate buffer ( $\mathrm{pH}$ 6.0) in a microwave oven. Thereafter, the samples were washed in deionized water, treated with $3 \%$ hydrogen peroxide, and washed in $0.01 \mathrm{M}$ phosphate-buffered saline (PBS; pH 7.4). Blocking was performed with $0.25 \%$ casein in $0.01 \mathrm{M}$ PBS and incubated overnight at $4{ }^{\circ} \mathrm{C}$ with the respective reagents.

For the detection of GM1 ganglioside, biotinylated cholera toxin B subunit (1:1000; List Biological Laboratories, Inc., Campbell, CA, USA) was used. For the detection of GM2 ganglioside, mouse anti-GM2 ganglioside monoclonal IgM antibody (1:1000; Tokyo Chemical Industry, Co., Ltd., Tokyo, Japan) was used as a primary antibody, and biotinylated goat anti-mouse IgM antibody (1:200; Vector Laboratories, Inc., Burlingame, CA, USA) was used as a secondary antibody. Subsequently, these sections were incubated with peroxidase-labeled streptavidin (KPL, Kirkegaard \& Perry Laboratories, Inc., Gaithersburg, MD, USA). The immunoreactivity was detected by a 3,3'-diaminobenzidine (DAB) system using DAB Tablet (Merck KGaA, Darmstadt, Germany) as a peroxidase substrate. The sections were counterstained with hematoxylin.

\section{Immunofluorescent study}

Each section was pretreated in the same way as described above for immunohistochemistry. For the detection of GM1 ganglioside, biotinylated cholera toxin B 
subunit (1:500; List Biological Laboratories, Inc.) and Alexa Fluor 488-conjugated streptavidin (1:1000; Life Technologies, Inc., Gaithersburg, MD, USA) were used. For the detection of GM2 ganglioside, mouse anti-GM2 monoclonal IgM antibody (1:500; Vector Laboratories, Inc.) was used as a primary antibody, and Alexa Fluor 488-conjugated goat anti-mouse IgM antibody (1:1000; Life Technologies, Inc.) was used as a secondary antibody. Subsequently, these sections were incubated with a 4',6-diamidino-2-phenylindole dihydrochloride (DAPI) solution (1:1000; Dojindo Laboratories, Inc., Kumamoto, Japan) for nuclear staining. The fluorescence was observed using a fluorescence microscopy (BX53-33-FL2, Olympus, Corp., Tokyo, Japan).

\section{Results}

Using the HE stain, cytoplasmic accumulation of pale to eosinophilic granular materials in balloon-swollen neurons was observed in the cerebral cortex samples of dogs and cats previously diagnosed with GM1 or GM2 gangliosidosis (Fig. 1a-d), whereas there was no such abnormal change observed in the samples of the control animals (Fig. 1e and f).

Using the immunohistochemical technique for the detection of GM1 ganglioside, the accumulated cytoplasmic materials were positively stained and mainly identified as GM1 ganglioside in cells of animals with confirmed GM1 gangliosidosis (Fig. 2a and b). In animals with GM2 gangliosidosis, the accumulated cytoplasmic materials were very weakly positively stained in a portion of the cells of the affected cat (Fig. 2c and d).
In the control animals, the cytoplasm in some normalshaped cells was also positively stained to indicate the presence of GM1 ganglioside (Fig. 2e and f). The nuclei of several cells were positively stained in a portion of the samples such as in the case of feline GM2 gangliosidosis and in both control animals (Fig. 2d-f).

Using the immunohistochemical technique for the detection of GM2 ganglioside, the accumulated cytoplasmic materials were positively stained and mainly identified as GM2 ganglioside in the cells of animals with GM2 gangliosidosis (Fig. 3c and d), whereas these materials were not strongly stained in animals with GM1 gangliosidosis (Fig. 3a and b). In the control animals, the cytoplasm in some normal-shaped cells was also weakly stained using this method (Fig. 3e and f). The nuclei of several cells were weakly positively stained in a portion of the samples such as in the case of canine GM1 gangliosidosis and in both control animals (Fig. 3a, e and $\mathrm{f}$ ).

The results of the immunofluorescent technique were almost identical to those of the immunohistochemical technique. The accumulated materials in the swollen neurons of animals with gangliosidoses were positively stained and clearly identified as either GM1 or GM2 ganglioside by using the respective detection techniques for each ganglioside (Figs. 4 and 5). The accumulated materials in the neurons of animals with GM2 gangliosidosis were very weakly stained using the technique for GM1 ganglioside (Fig. 4c and d), and vice versa (Fig. 5a and $\mathrm{b})$. In the control animals, some cells showed

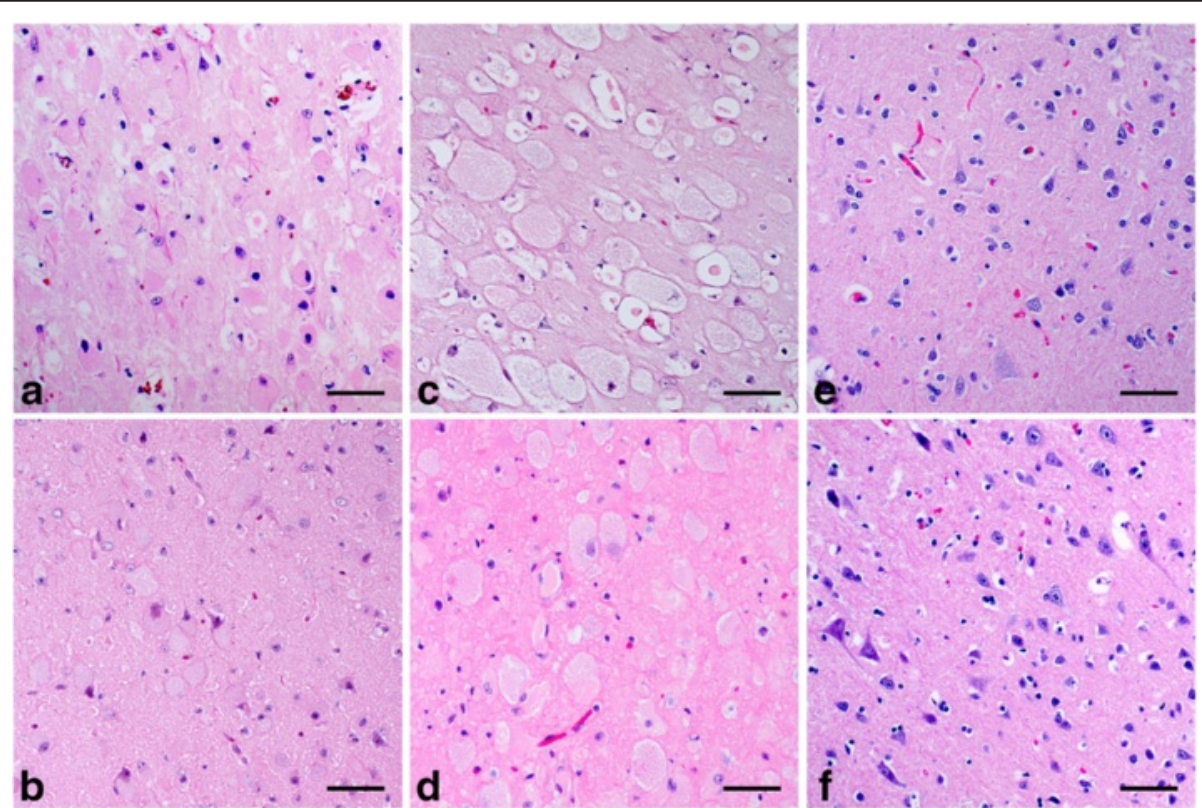

Fig. 1 Histopathological findings in animals affected and unaffected with gangliosidoses. Hematoxylin and eosin staining was performed on paraffin-embedded sections of the cerebral cortex from the following animals: a dog (a) and a cat (b) affected with GM1 gangliosidosis; a dog (c) and a cat (d) affected with GM2 gangliosidosis; an unaffected control dog (e) and cat (f). Bar $=50 \mu \mathrm{m}$ 


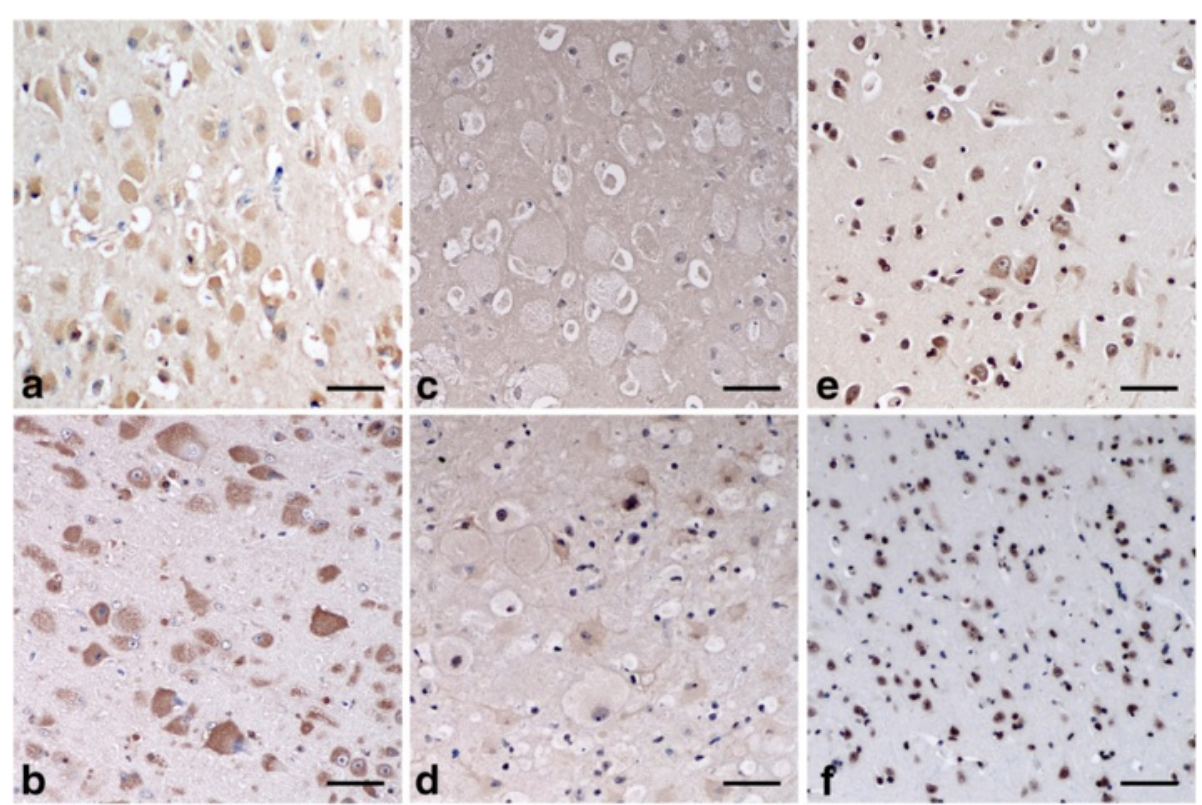

Fig. 2 Immunohistochemical findings for the detection of GM1 ganglioside in animals affected and unaffected with gangliosidoses. The immunohistochemical technique for the detection of GM1 ganglioside was performed on paraffin-embedded sections of the cerebral cortex from the following animals: a dog (a) and a cat (b) affected with GM1 gangliosidosis; a dog (c) and a cat (d) affected with GM2 gangliosidosis; an unaffected control dog (e) and cat (f). For the detection of GM1 ganglioside, biotinylated cholera toxin B subunit and peroxidase-labeled streptavidin were used. The immunoreactivity was detected by 3,3'-diaminobenzidine as a peroxidase substrate. The sections were counterstained with hematoxylin. Bar $=50 \mu \mathrm{m}$

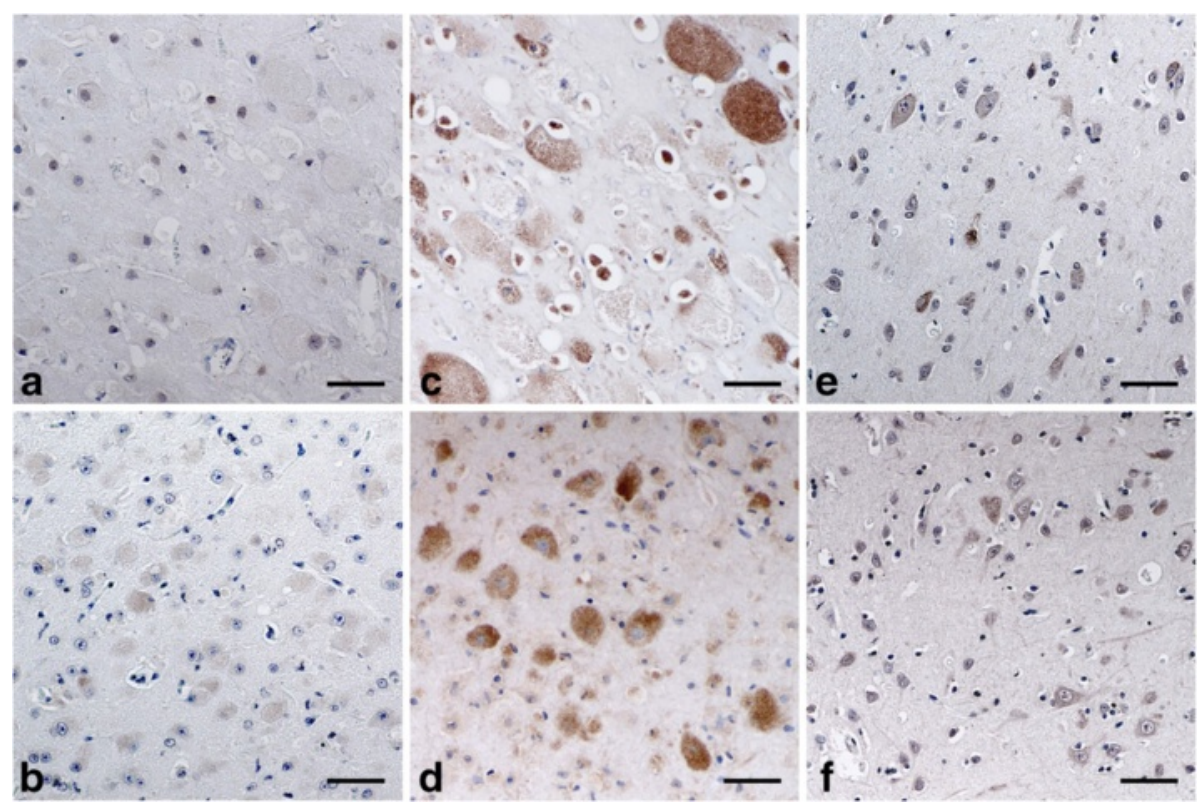

Fig. 3 Immunohistochemical findings for the detection of GM2 ganglioside in animals affected and unaffected with gangliosidoses. The immunohistochemical technique for the detection of GM2 ganglioside was performed on paraffin-embedded sections of the cerebral cortex from the following animals: a dog (a) and a cat (b) affected with GM1 gangliosidosis; a dog (c) and a cat (d) affected with GM2 gangliosidosis; an unaffected control dog (e) and cat (f). For the detection of GM2 ganglioside, mouse anti-GM2 ganglioside monocloncal IgM antibody was used as a primary antibody, and biotinylated goat anti-mouse IgM antibody was used as a secondary antibody. Subsequently, these sections were incubated with peroxidase-labeled streptavidin. The immunoreactivity was detected by 3,3'-diaminobenzidine as a peroxidase substrate. The sections were counterstained with hematoxylin. Bar $=50 \mu \mathrm{m}$ 

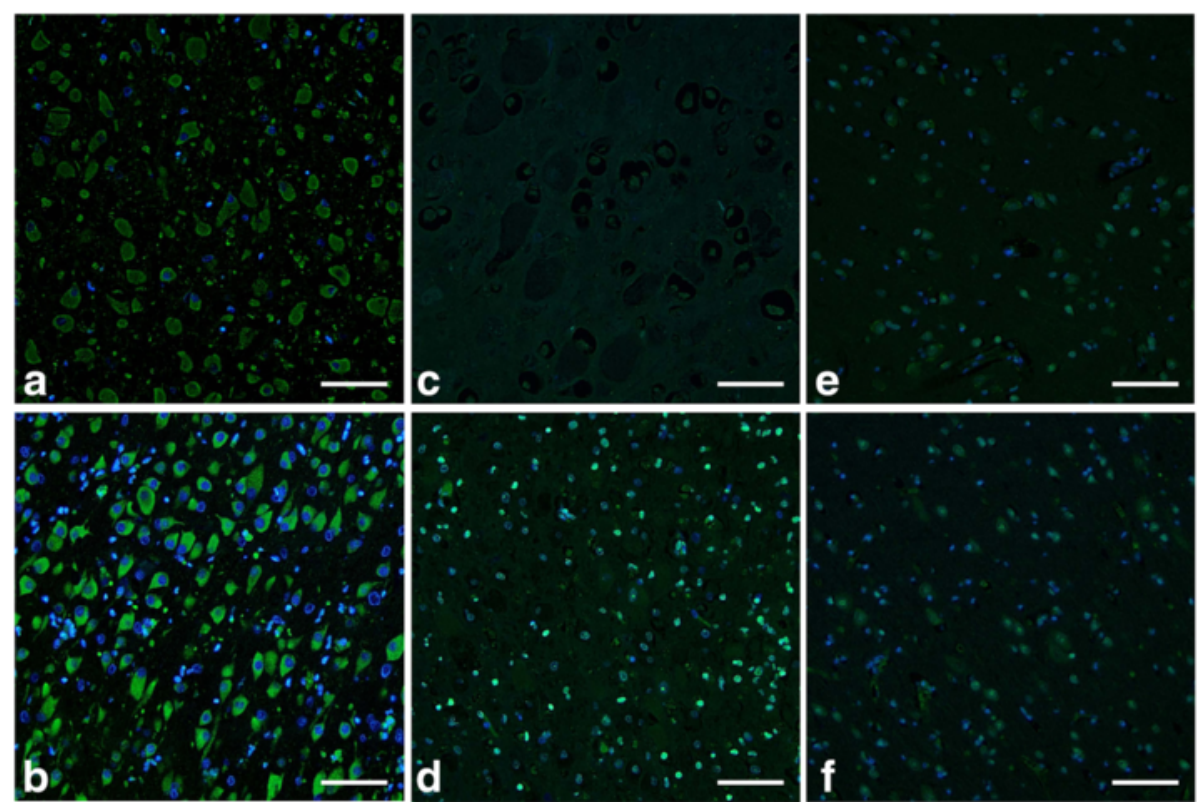

Fig. 4 Immunofluorescent findings for the detection of GM1 ganglioside in animals affected and unaffected with gangliosidoses. The immunofluorescent technique for the detection of GM1 ganglioside was performed on paraffin-embedded sections of the cerebral cortex from the following animals: a dog (a) and a cat (b) affected with GM1 gangliosidosis; a dog (c) and a cat (d) affected with GM2 gangliosidosis; an unaffected control dog (e) and cat (f). For the detection of GM1 ganglioside, biotinylated cholera toxin B subunit and Alexa Fluor 488-conjugated streptavidin were used. Subsequently, these sections were incubated with 4',6-diamidino-2-phenylindole dihydrochloride for nuclear staining. Bar $=30 \mu \mathrm{m}$

cytoplasm that was positively stained for GM1 and GM2 gangliosides (Figs. 4 and $5 \mathrm{e}$ and $\mathrm{f}$ ). In addition, some of the cells of a cat with GM2 gangliosidosis and both control animals showed positive staining of nuclei using the technique for the detection of GM1 ganglioside (Fig. 4d-f). Some of the cells of a control cat showed weakly positive staining of nuclei using the technique for the detection of GM2 ganglioside (Fig. 5f).

\section{Discussion}

Gangliosides are glycosphingolipids consisting of a hydrophobic ceramide ( $N$-acylsphingosine) and a hydrophilic oligosaccharide chain bearing one or more $\mathrm{N}$-acetylneuraminic acid (silalic acid) residues, and are typical components of the outer leaflet of the plasma membranes of animal cells [2, 3]. GM1 and GM2 gangliosides are present as the main glycolipids in neurons and are likely to be involved in cell differentiation and cell-cell interactions, but their specific physiological functions remain obscure. Therefore, developing techniques for the detection of GM1 and GM2 gangliosides is important not only for advancement in brain science but also for the correct diagnosis of gangliosidoses, because the intralysosomal accumulation of each ganglioside in neurons is characteristic to either GM1 or GM2 gangliosidosis. Therefore, in the past few decades, various determination methods for the profiling, quantification, or evaluation of gangliosides, including GM1 and GM2 gangliosides, in tissues, cultured cells, or extracellular fluids have been reported. These methods include TLC coupled with densitometric or immunochemical detection [43, 47], high-performance liquid chromatography coupled with tandem mass spectrometric detection [48], enzyme-linked immunosorbent assay [49], and matrixassisted laser desorption ionization time-of-flight mass spectrometry [50].

The in situ detection of gangliosides in tissue sections is also very important not only for diagnosis of the diseases but also to obtain reliable information on their tissue, cellular, and subcellular distributions [51]. Furthermore, confirming that the histological detection of GM1 and GM2 gangliosides is applicable to paraffinembedded specimens stored for a long period would also be useful for the retrospective diagnosis of the diseases, but very few studies have evaluated such in situ detection methods using long-term stored paraffin-embedded specimens from canine and feline gangliosidoses. In the present study, immunohistochemical and immunofluorescent techniques for the detection of GM1 and GM2 gangliosides were developed, and their application was evaluated using canine and feline paraffin-embedded specimens stored for 5 to 11 years. As a result, these two techniques could clearly detect the presence of both GM1 and GM2 gangliosides in neurons of the control animals (Figs. 2, 3, 4 and 5e and f) as well as the accumulation of either GM1 or GM2 ganglioside in neurons 


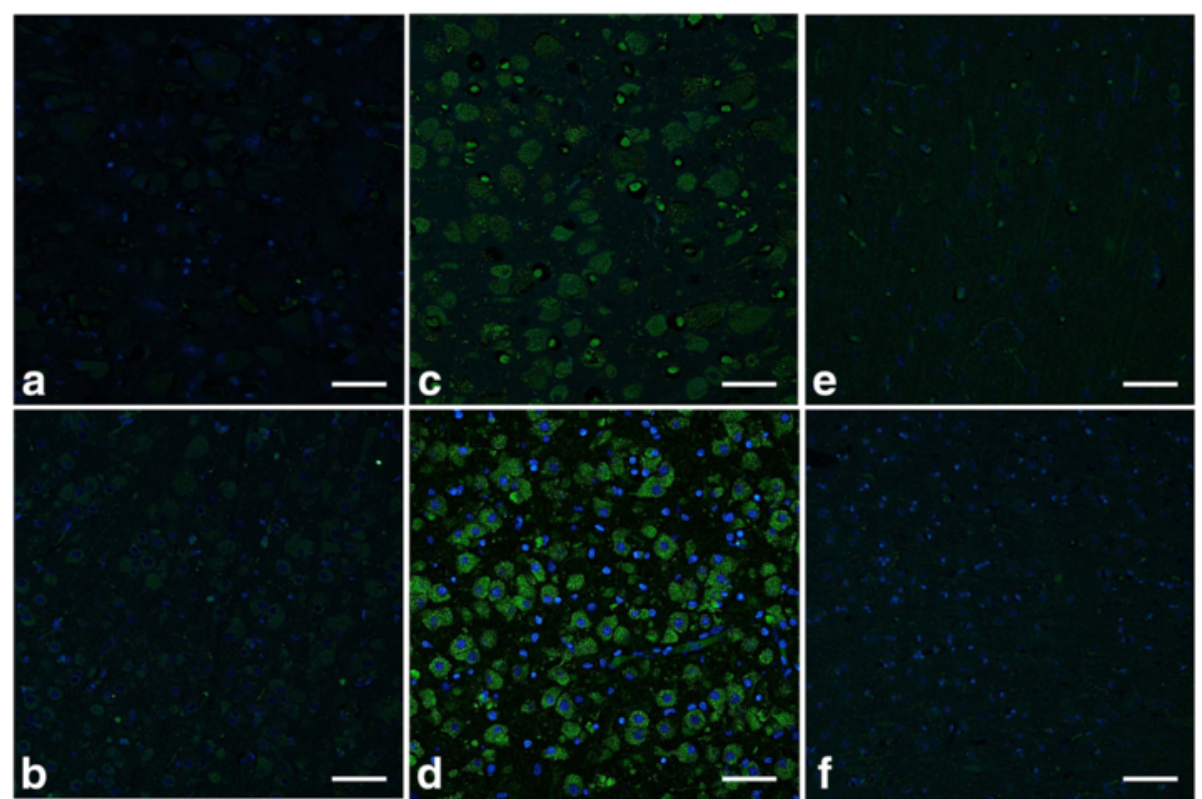

Fig. 5 Immunofluorescent findings for the detection of GM2 ganglioside in animals affected and unaffected with gangliosidoses. The immunofluorescent technique for the detection of GM2 ganglioside was performed on paraffin-embedded sections of the cerebral cortex from the following animals: a dog (a) and a cat (b) affected with GM1 gangliosidosis; a dog (c) and a cat (d) affected with GM2 gangliosidosis; an unaffected control dog (e) and cat (f). For the detection of GM2 ganglioside, mouse anti-GM2 monoclonal lgM antibody was used as a primary antibody, and Alexa Fluor 488-conjugated goat anti-mouse IgM antibody was used as a secondary antibody. Subsequently, these sections were incubated with 4',6diamidino-2-phenylindole dihydrochloride for nuclear staining. Bar $=30 \mu \mathrm{m}$

of animals with diagnosed GM1 (Figs. 2, 3, 4 and 5a and b) and GM2 gangliosidoses (Figs. 2, 3, 4 and $5 \mathrm{c}$ and d). These data demonstrate that the two techniques are applicable to the retrospective in situ detection of GM1 and GM2 gangliosides, and consequently to the auxiliary diagnosis of gangliosidoses in dogs and cats. However, gangliosides can be accumulated as the secondary products without direct link to the primary protein defect in some lysosomal and a few non-lysosomal diseases [2, 52]. Therefore, in cases in which the abnormal accumulation of each ganglioside is found in swollen neurons, a definitive diagnosis should ultimately be made using DNA extracted from the same paraffin-embedded specimen via the identification of pathogenic mutation(s) in the responsible genes: the GLB1 gene for GM1 gangliosidosis and the HEXA, HEXB, and GM2A genes for GM2 gangliosidosis.

Comparing the two techniques developed in the present study, the immunofluorescent technique provided relatively less histopathological information than the immunohistochemical technique, due to the dark background when using immunofluorescence. Therefore, the tissue, cellular, and subcellular distributions of stained materials could not be easily determined in the immunofluorescent technique; however, this technique does have the advantage of requiring a lower amount of reagents (nearly half) because of its higher detection sensitivity compared to the immunohistochemical technique.

In addition, in the experiments conducted to detect GM1 and GM2 gangliosides, the nuclei were stained in some specimens using both techniques. The positive staining of the nuclei in some cells from affected and control animals may result from the natural components of GM1 and GM2 gangliosides because the nuclei of neuronal cells in rat brain contain these gangliosides [53]. However, this stain of the nucleus was easily differentiated from the specific stain of cytoplasmic GM1 and GM2 gangliosides when using the immunohistochemical technique but not when using the immunofluorescent technique, owing to the reduced morphological visibility. Therefore, the simultaneous observation of HE-stained cerebral tissues (Fig. 1) is necessary for accurate judgment of the results, especially when using an immunofluorescent technique.

\section{Conclusions}

The immunohistochemical and immunofluorescent techniques for the detection of GM1 and GM2 gangliosides established in this study are useful for the auxiliary diagnosis of GM1 and GM2 gangliosidoses in dogs and cats before a definitive diagnosis can be made using molecular analysis for identification of causative mutations. 
These techniques may also be useful for the retrospective diagnosis of suspected cases of all animal species for which paraffin-embedded cerebral tissues are stored.

\section{Abbreviations}

DAB: 3,3'-diaminobenzidine; DAPI: 4',6-diamidino-2-phenylindole dihydrochloride; HE: hematoxylin and eosin; PBS: phosphate-buffered saline: TLC: thin-layer chromatography.

\section{Competing interests}

The authors declare that they have no competing interests.

\section{Authors' contributions}

MK carried out the histopathological, immunohistochemical, and immunofluorescent studies and drafted the manuscript. AY participated in the design of the study. KO, YN, KU, DH, KT, HK, and MT prepared the paraffin-embedded tissues samples for the original diagnosis of animals used in this study. OY supervised the project, designed the study, and reviewed the results and manuscript. All authors read and approved the final version of the manuscript.

\section{Acknowledgements}

This study was supported financially by grants (25292181 and 26660242 OY) from the Ministry of Education, Culture, Sports, Science and Technology of Japan.

\section{Author details}

${ }^{1}$ Laboratory of Clinical Pathology, Department of Veterinary Medicine, Joint Faculty of Veterinary Medicine, Kagoshima University, 1-21-24 Kohrimoto, Kagoshima-shi, Kagoshima 890-0065, Japan. ${ }^{2}$ Laboratory of Veterinary Pathology, Department of Veterinary Medicine, Faculty of Agriculture, Iwate University, 3-18-8 Ueda, Morioka-shi, Iwate 020-8550, Japan. ${ }^{3}$ Kyoto Animal Referral Medical Center, 208-4 Shin-arami, Tai, Kumiyama-cho, Kuse-gun, Kyoto 613-0036, Japan. ${ }^{4}$ Laboratory of Veterinary Pathology, Graduate School of Agricultural and Life Sciences, The University of Tokyo, 1-1-1 Yayoi, Bunkyou-ku, Tokyo 113-8657, Japan. ${ }^{5}$ Department of Veterinary Radiology, Nippon Veterinary and Life Science University, 1-7-1 Kyouman-chou, Musashino-shi, Tokyo 180-8602, Japan. 'Department of Veterinary Pathology, Nippon Veterinary and Life Science University, 1-7-1 Kyouman-chou, Musashino-shi, Tokyo, 180-8602, Japan. ' Laboratory of Veterinary Histopathology, Department of Veterinary Medicine, Joint Faculty of Veterinary Medicine, Kagoshima University, 1-21-24 Kohrimoto, Kagoshima-shi, Kagoshima 890-0065, Japan.

\section{Received: 31 December 2015 Accepted: 22 March 2016} Published online: 31 March 2016

\section{References}

1. Haskins M, Giger U. Lysosomal storage diseases. In: Kaneko JJ, Harvey JW, Bruss ML, editors. Clinical biochemistry of domestic animals. 6th ed. Burlington: Academic; 2008. p. 731-50.

2. Suzuki Y, Oshima A, Nanba E. $\beta$-Galactosidase deficiency (B-galactosidosis): GM1 gangliosidosis and Morquio B disease. In: Scriver CR, Beaudet AL, Sly WS, Valle $D$, editors. The metabolic and molecular bases of inherited disease. 8th ed. New York: McGraw-Hill; 2001. p. 3775-809.

3. Gravel RA, Kaback MM, Proia RL, Sandhoff K, Suzuki K. The GM2 gangliosidosis. In: Scriver CR, Beaudet AL, Sly WS, Valle D, editors. The metabolic and molecular bases of inherited disease. 8th ed. McGraw-Hill: New York; 2001. p. 3824-76.

4. Read DH, Harrington DD, Keenana TW, Hinsman EJ. Neuronal-visceral GM1 gangliosidosis in a dog with beta-galactosidase deficiency. Science. 1976;194:442-5

5. Alroy J, Orgad U, Ucci AA, Schelling SH, Schunk KL, Warren CD, et al. Neurovisceral and skeletal GM1-gangliosidosis in dogs with betagalactosidase deficiency. Science. 1985;229:470-2.

6. Saunders GK, Wood PA, Myers RK, Shell LG, Carithers R. GM1 gangliosidosis in Portuguese water dogs: pathologic and biochemical findings. Vet Pathol. 1988;25:265-9.

7. Müller G, Baumgärtner W, Moritz A, Sewell A, Kustermann-Kuhn B. Biochemical findings in a breeding colony of Alaskan huskies suffering from GM1-gangliosidosis. J Inherit Metab Dis. 1998;21:430-1.
8. Yamato O, Ochiai K, Masuoka Y, Hayashida E, Tajima M, Omae S, et al. GM1 gangliosidosis in shiba dogs. Vet Rec. 2000;146:493-6.

9. Whitfield P, Johnson AW, Dunn KA, Delauche AJN, Winchester BG, Franklin RJM. GM1-gangliosidosis in a cross-bred dog confirmed by detection of GM1-ganglioside using electrospray ionisation-tandem mass spectrometry. Acta Neuropathol. 2000;100:409-14.

10. Baker Jr HJ, Lindsey JR, McKhann GM, Farrell DF. Neuronal GM1 gangliosidosis in a Siamese cat with $\beta$-galactosidase deficiency. Science. 1971;174:838-9.

11. Uddin MM, Tanimoto T, Yabuki A, Kotani T, Kuwamura M, Chang HS, et al. Mutation analysis of GM1 gangliosidosis in a Siamese cat from Japan in the 1960s. J Feline Med Surg. 2012;14:900-2.

12. De Maria R, Divari S, Bo S, Sonnio S, Lotti D, Capucchio MT, et al. $\beta$ Galactosidase deficiency in a Korat cat: a new form of feline GM1 gangliosidosis. Acta Neuropathol. 1998;96:307-14.

13. Blakemore WF. GM1 gangliosidosis in a cat. J Comp Pathol. 1972;82:179-85.

14. Barnes IC, Kelly DF, Pennock CA, Randell DJ. Hepatic beta galactosidase and feline GM1 gangliosidosis. Neuropathol Appl Neurobiol. 1981;7:463-76.

15. Baker CG, Blakemore WF, Dell A, Palmer AC, Tiller PR, Winchester BG. GM1 gangliosidosis (type 1) in a cat. Biochem J. 1986;235:151-8.

16. Uddin MM, Hossain MA, Rahman MM, Chowdhury MA, Tanimoto T, Yabuki A, et al. Identification of Bangladesh domestic cats with GM1 gangliosidosis caused by the $c .1448 \mathrm{G}>\mathrm{C}$ mutation of the feline GLB1 gene: case study. J Vet Med Sci. 2013;75:395-7.

17. Ueno H, Yamato O, Sugiura T, Kohyama M, Yabuki A, Miyoshi K, et al. GM1 gangliosidosis in a Japanese domestic cat: a new variant identified in Hokkaido, Japan. J Vet Med Sci. 2016;78:91-5.

18. Donnelly WJ, Sheahan BJ, Kelly M. Beta-galactosidase deficiency in GM1 gangliosidosis of Friesian calves. Res Vet Sci. 1973;15:139-41.

19. Ahern-Rindell AJ, Prieur DJ, Murname RD, Raghavan SS, Daniel PF, McCluer RH, et al. Inherited lysosomal storage disease associated with deficiencies of beta-galactosidase and alfa-neuraminidase in sheep. Am J Med Genet. 1988;31:39-56.

20. Skelly BJ, Jeffrey M, Franklin RJM, Winchester BG. A new form of ovine GM1gangliosidosis. Acta Neuropathol. 1995;89:374-9.

21. Ryder SJ, Simmons MM. A lysosomal storage disease of Romney sheep that resembles human type 3 GM1 gangliosidosis. Acta Neuropathol. 2001;101: 225-8.

22. Muthupalani S, Torres PA, Wang BC, Zeng BJ, Eaton S, Erdelyi I, et al. GM1gangliosidosis in American black bears: clinical, pathological, biochemical and molecular genetic characterization. Mol Genet Metab. 2014;111:513-21.

23. Bermudez AJ, Johnson GC, Vanier MT, Schröder M, Suzuki K, Stogsdill PL, et al. Gangliosidosis in emus (Dromaius novaehollandiae). Avian Dis. 1995;39: 292-303.

24. Karbe E. Animal model: canine GM2-gangliosidosis. Am J Pathol. 1973; 71:151-4.

25. Cummings JF, Wood PA, Walkley SU, de Lahunta A, DeForest ME. GM2 gangliosidosis in a Japanese spaniel. Acta Neuropathol. 1985;67:247-53.

26. Sanders DN, Zeng R, Wenger DA, Johnson GS, Johnson GC, Decker JE, et al. GM2 gangliosidosis associated with a HEXA missense mutation in Japanese Chin dogs: a potential model for Tay Sachs disease. Mol Genet Metab. 2013;108:70-5.

27. Yamato O, Matsuki N, Satoh H, Inaba M, Ono K, Yamasaki M, et al. Sandhoff disease in a golden retriever dog. J Inherit Metab Dis. 2002;25:319-20.

28. Tamura S, Tamura Y, Uchida K, Nibe K, Nakaichi M, Hossain MA, et al. GM2 gangliosidosis variant 0 (Sandhoff-like disease) in a family of toy poodles. J Vet Intern Med. 2010;24:1013-9.

29. Rotmistrovsky RA, Alcaraz A, Cummings JC, de Lahunta A, Farmer SF. GM2 gangliosidosis in a mix-breed dog. Prog Vet Neurol. 1991;2:203-8.

30. Kohyama M, Yabuki A, Kawasaki Y, Miura N, Kitano Y, Onitsuka T, et al. GM2 gangliosidosis variant 0 (Sandhoff disease) in a mixed-breed dog. J Am Anim Hosp Assoc. 2015;51:396-400.

31. Neuwelt EA, Johnson WG, Blank NK, Pagel MA, Masien-McClure C, McClure $\mathrm{MJ}$, et al. Characterization of a new model of GM2-gangliosidosis (Sandhoff's disease) in Korat cats. J Clin Invest. 1985;76:482-90.

32. Bradbury AM, Morrison NE, Hwang M, Cox NR, Baker HJ, Martin DR. Neurodegenerative lysosomal storage disease in European Burmese cats with hexosaminidase $\beta$-subunit deficiency. Mol Genet Metab. 2009;97:53-9.

33. Cork LC, Munnell JF, Lorenz MD, Murphy JV, Baker HJ, Rattazzi MC. GM2 ganglioside lysosomal storage disease in cats with $\beta$-hexosaminidase deficiency. Science. 1977;196:1014-7. 
34. Yamato O, Matsunaga S, Takata K, Uetsuka K, Satoh H, Shoda T, et al. GM2gangliosidosis variant 0 (Sandhoff-like disease) in a family of Japanese domestic cats. Vet Rec. 2004;155:739-44.

35. Martin DR, Cox NR, Morrison NE, Kennamer DM, Peck SL, Dodson AN, et al. Mutation of the GM2 activator protein in a feline model of GM2 gangliosidosis. Acta Neuropathol. 2005;110:443-50.

36. Kosanke SD, Pierce KR, Bay WW. Clinical and biochemical abnormalities in porcine GM2 gangliosidosis. Vet Pathol. 1978;15:685-99.

37. Torres PA, Zeng BJ, Porter BF, Alroy J, Horak F, Horak J, et al. Tay-Sachs disease in Jacob sheep. Mol Genet Metab. 2010;101:357-63.

38. Rickmeyer T, Schöniger S, Petermann A, Harzer K, Kustermann-Kuhn B, Fuhrmann $\mathrm{H}$, et al. GM2 gangliosidosis in an adult pet rabbit. J Comp Pathol. 2013;148:243-7.

39. Fox J, Li YT, Dawson G, Alleman A, Johnsrude J, Schumacher J, et al. Naturally occurring GM2 gangliosidosis in two Muntjak deer with pathological and biochemical features of human classical Tay-Sachs disease (type B GM2 gangliosidosis). Acta Neuropathol. 1999;97:57-62.

40. Zeng BJ, Torres PA, Viner TC, Wang ZH, Raghavan SS, Alroy J, et al. Spontaneous appearance of Tay-Sachs disease in an animal model. Mol Genet Metab. 2008;95:59-65.

41. Hasegawa D, Tamura S, Nakamoto Y, Matsuki N, Takahashi K, Fujita M, et al. Magnetic resonance findings of the corpus callosum in canine and feline lysosomal storage diseases. PLoS One. 2013;8(12):e0083455.

42. Yamato $O$, Kobayashi $A$, Satoh $H$, Endoh D, Shoda T, Masuoka Y, et al. Comparison of polymerase chain reaction-restriction fragment length polymorphism assay and enzyme assay for diagnosis of GM1-gangliosidosis in Shiba dogs. J Vet Diagn Invest. 2004;16:299-304.

43. Yamato O, Satoh H, Matsuki N, Ono K, Yamasaki M, Maede Y. Laboratory diagnosis of canine GM2-gangliosidosis using blood and cerebrospinal fluid. J Vet Diagn Invest. 2004;16:39-44.

44. Chang HS, Arai T, Yabuki A, Hossain MA, Rahman MM, Mizukami K, et al. Rapid and reliable genotyping technique for GM1 gangliosidosis in Shiba dogs by real-time polymerase chain reaction with TagMan minor groove binder probes. J Vet Diagn Invest. 2010;22:234-7.

45. Rahman MM, Yabuki A, Kohyama M, Mitani S, Mizukami K, Uddin MM, et al. Real-time PCR genotyping assay for GM2 gangliosidosis variant 0 in Toy Poodles and the mutant allele frequency in Japan. J Vet Med Sci. 2014;76:295-9.

46. Rahman MM, Shoubudani T, Mizukami K, Chang HS, Hossain MA, Yabuki A, et al. Rapid and simple polymerase chain reaction-based diagnostic assays for GM2 gangliosidosis variant 0 (Sandhoff-like disease) in Japanese domestic cats. J Vet Diagn Invest. 2011;23:338-42.

47. Satoh H, Yamato O, Asano T, Yamasaki M, Maede Y. Increased concentration of GM1-ganglioside in cerebrospinal fluid in dogs with GM1- and GM2-gangliosidoses and its clinical application for diagnosis. J Vet Diagn Invest. 2004;16:223-6.

48. Huang Q, Zhou X, Liu D, Xin B, Cechner K, Wang H, Zhou A. A new liquid chromatography/tandem mass spectrometry method for quantification of gangliosides in human plasma. Anal Biochem. 2014;455:26-34.

49. Dawson RM. Characterization of the binding of cholera toxin to ganglioside GM1 immobilized onto microtitre plates. J Appl Toxicol. 2005;25:30-8.

50. Satoh H, Yamauchi T, Yamasaki M, Maede Y, Yabuki A, Chang HS, et al. Rapid detection of GM1 ganglioside in cerebrospinal fluid in dogs with GM1 gangliosidosis using matrix-assisted laser desorption ionization timeof-flight mass spectrometry. J Vet Diagn Invest. 2011;23:1202-7.

51. Petr T, Šmíd V, Šmídová J, Hůlková H, Jirkovská M, Elleder M, et al. Histochemical detection of GM1 ganglioside using cholera toxin-B subunit. Evaluation of critical factors optimal for in situ detection with special emphasis to acetone pre-extraction. Eur J Histochem. 2010;54:e23.

52. Walkley SU, Vanier MT. Secondary lipid accumulation in lysosomal disease. Biochim Biophys Acta. 2009:1793:726-36.

53. Saito M, Sugiyama K. Characterization of nuclear gangliosides in rat brain: concentration, composition, and developmental changes. Arch Biochem Biophys. 2002;398:153-9.

\section{Submit your next manuscript to BioMed Central and we will help you at every step:}

- We accept pre-submission inquiries

- Our selector tool helps you to find the most relevant journal

- We provide round the clock customer support

- Convenient online submission

- Thorough peer review

- Inclusion in PubMed and all major indexing services

- Maximum visibility for your research

Submit your manuscript at www.biomedcentral.com/submit
C) Biomed Central 